\title{
FASTSIM2: a second-order accurate frictional rolling contact algorithm
}

\author{
E. A. H. Vollebregt • P. Wilders
}

Received: 23 June 2010 / Accepted: 17 August 2010 / Published online: 4 September 2010

(C) The Author(s) 2010. This article is published with open access at Springerlink.com

\begin{abstract}
In this paper we consider the frictional (tangential) steady rolling contact problem. We confine ourselves to the simplified theory, instead of using full elastostatic theory, in order to be able to compute results fast, as needed for on-line application in vehicle system dynamics simulation packages. The FASTSIM algorithm is the leading technology in this field and is employed in all dominant railway vehicle system dynamics packages (VSD) in the world. The main contribution of this paper is a new version "FASTSIM2" of the FASTSIM algorithm, which is second-order accurate. This is relevant for VSD, because with the new algorithm 16 times less grid points are required for sufficiently accurate computations of the contact forces. The approach is based on new insights in the characteristics of the rolling contact problem when using the simplified theory, and on taking precise care of the contact conditions in the numerical integration scheme employed.
\end{abstract}

Keywords Frictional rolling contact · Fastsim algorithm • Wheel-rail contact $\cdot$ Numerical integration scheme

\section{Introduction}

The dynamic behavior and stability of railway vehicles strongly depends on the wheel/rail interaction. Therefore, railway vehicle system dynamics (VSD) analysis tools

E. A. H. Vollebregt $(\varangle) \cdot$ P. Wilders

DIAM, Delft University of Technology, P.O. Box 5031,

2600 GA Delft, The Netherlands

e-mail: e.a.h.vollebregt@tudelft.nl

E. A. H. Vollebregt

VORtech, P.O. Box 260, 2600 AG Delft, The Netherlands include a contact model in one form or another [1]. For the normal problem a Hertzian approach is typically employed $[2,3]$, and extensions to non-Hertzian geometries are also available $[4,5]$. For the tangential creep force and torsional moment different contact theories are available, see $[3,6,7]$ for different overviews.

Many theories with respect to the rolling contact problem for railway applications are due to Prof. J.J. Kalker of Delft University of Technology. Particularly the variational approach implemented in his program CONTACT [7-9] is regarded as an exact theory. However, this program is in many cases too slow for use in VSD packages, in which millions or billions of contact problems must be solved. Therefore currently, these packages have to rely on approximations of one or another sort.

In this paper we concentrate on the FASTSIM algorithm [10] that is based on the so-called simplified theory [11], where the material constitutive behaviour is schematised. This algorithm is used in many VSD packages, because it is fast and it has an attractive foundation, e.g. see [12]. It is also used in many research studies on the wheel rail interface $[13,14]$ or even for the calculation of wear [15], where the more accurate CONTACT approach (or finite element approaches like that of [16]) would be preferred if it were computationally feasible. Extensions of the original FASTSIM algorithm have been proposed in literature for non-Hertzian geometries [17], for more generic friction laws $[18,19]$, and for the computation of unsteady contact as well $[12,20]$.

The total tangential forces computed with FASTSIM are typically accurate up to $5 \%$ when compared to the CONTACT results, although errors larger than $20 \%$ are found regularly as well, particularly in cases with a large amount of spin creepage [21]. To achieve these results requires that a sufficiently fine grid discretisation is used, otherwise discretisation errors 
may be leading and spoil the accuracy. However, to use a fine discretisation implies an increase of the computational time.

In this paper we show that FASTSIM's original discretisation approach can be viewed as the backward Euler integration rule and is only first-order accurate. We analyze a 1D model problem in order to gain insight in the structure of solutions and to construct new numerical techniques. We then propose a new approach, called FASTSIM2, that is based on the trapezoidal rule and that is second-order accurate. Finally, we demonstrate the grid convergence of the new approach and show how few elements are needed to make discretisation errors small compared to FASTSIM's theoretical accuracy.

A related work in the area of finite element contact modeling is presented in [16]. A marked difference to that work is that FASTSIM employs a schematised material model such that material points are independent of each other. The FASTSIM approach amounts to the method of characteristics [22], in which the time-evolution for a single material particle is solved. This corresponds to an "Unsplit" strategy in the terminology of [16], using a Lagrangian view, and avoids the need for a numerical advection scheme.

This paper is structured as follows. In Section 2 we present the formulation of the contact problem and the original FASTSIM algorithm. Section 3 develops the main ideas underlying FASTSIM2 using a 1D model study. Section 4 describes the FASTSIM2 algorithm itself. Finally, Section 5 describes the results obtained in our experiments.

\section{Formulation of the contact problem}

This section is largely based on [7] and for background information the reader is referred to this book.

\subsection{Nomenclature, geometry}

We consider two elastic bodies, particularly a wheel and a rail. We first identify their geometry without considering deformations, the so-called undeformed reference state. Particles of the bodies are referred to by their coordinates $\mathbf{x}$ in the coordinate system $O x y z$ used in this reference state. The right-hand coordinate system is assumed to be moving with the contact area, with the $x$-axis pointing in the rolling direction and $z$ pointing upwards, into body 1 , the wheel.

The bodies are brought into contact and, as a result, stresses $\sigma$, strains $\boldsymbol{\epsilon}$ and displacements $\mathbf{u}$ arise in the bodies and at their surfaces. In formulating the contact problem, we are particularly interested in the surface quantities: the displacements $\mathbf{u}^{(a)}(\mathbf{x})$ of the surface particles $\mathbf{x}$ of bodies $a=1,2$ and the surface tractions (stresses) $\mathbf{p}^{(a)}(\mathbf{x})$. Now, since $\mathbf{p}^{(2)}(\mathbf{x})=-\mathbf{p}^{(1)}(\mathbf{x})$ for all surface positions $\mathbf{x}$ where the bodies are in contact, we may eliminate $\mathbf{p}^{(2)}$ and consider a single variable $\mathbf{p}(\mathbf{x})=\mathbf{p}^{(1)}(\mathbf{x})$ for the contact area. Furthermore, the displacements enter the contact problem mainly through their differences. Therefore, we introduce the so-called displacement difference $\mathbf{u}(\mathbf{x})$ at position $\mathbf{x}: \mathbf{u}(\mathbf{x})=\mathbf{u}^{(1)}(\mathbf{x})-\mathbf{u}^{(2)}(\mathbf{x})$.

The quantities introduced above are tensors $(\boldsymbol{\sigma}, \boldsymbol{\epsilon})$ and vectors $(\mathbf{x}, \mathbf{u}, \mathbf{p})$ in three-dimensional space. In this paper we concentrate on the tangential contact problem. In the remainder we will therefore distinguish between scalar quantities of the normal contact problem, and vectors of two components, corresponding to the coordinate directions $x$ and $y$. The normal coordinate direction is often indicated with subscript $n$. The tangential 2-vectors are denoted by boldface symbols, the tangential directions are indicated by $\tau=x, y$. Subscripts $i, j$ are used for grid point numbers in $x$ - and $y$-directions.

\subsection{The contact conditions}

In the normal problem a significant role is played by the deformed distance $e$ :

$e:=h+u_{n}$.

Here, $h$ is the distance between the bodies' surfaces in the normal direction in the undeformed state. For the tangential problem, an important quantity is the (relative) slip $\mathbf{s}:^{1}$

$\mathbf{s}:=\mathbf{w}+\dot{\mathbf{u}} / V$.

Here, (dot) denotes the particle-fixed, material time derivative. The quantity $\dot{\mathbf{u}}$ describes the rate of change of the displacement difference of two opposing particles of bodies 1 and 2. $V$ is the rolling velocity. Finally, $\mathbf{w}$ is the relative rigid slip, i.e. the mutual velocity of the contacting particles within the undeformed reference system, relative to the rolling velocity $V$. In concentrated contact problems this is given by

$\mathbf{w}=[\xi-\phi y, \eta+\phi x]^{T}$.

$\xi$ and $\eta$ are the longitudinal and lateral creepage, respectively. $\phi$ is the spin creepage, representing the relative rotation of the bodies around the $z$-axis.

With these quantities, the following contact conditions are defined:

normal problem :

in exterior area $E: \quad e>0, p_{n}=0$

in contact $C=H \cup S: \quad e=0, p_{n} \geq 0$

tangential problem :

in exterior area $E: \quad \mathbf{s}$ free, $\mathbf{p}=\mathbf{0}$,

in adhesion area $H: \quad\|\mathbf{s}\|=0,\|\mathbf{p}\| \leq g$,

in slip area $S$ :

$$
\|\mathbf{s}\|>0, \mathbf{p}=-g \mathbf{s} /\|\mathbf{s}\|
$$

${ }^{1}$ Unfortunately, the definitions of $\mathbf{s}$ and $\mathbf{w}$ are interchanged in the original FASTSIM paper [10]. We adhere to the notations used in [7] and in more recent work. 
Equations (4) and (5) state that the normal pressure is compressive and there is no interpenetration between the bodies. Equations (7) and (8) state that the tangential tractions cannot exceed the so-called traction bound $g$, that slip occurs only where $\mathbf{p}$ is at the traction bound, and that the tractions then act in the opposite direction to the slip.

Different formulations are possible for the traction bound $g$. In this paper we use Coulomb's friction law, applied locally, with (static) coefficient of friction $\mu$ :

$g=\mu p_{n}$

\subsection{Kalker's variational CONTACT approach}

In the presentation above we have ignored the relations between surface tractions $\mathbf{p}$ and displacements $\mathbf{u}$. These depend on the constitutive equations that describe the material behaviour, as well as on the geometries of the bodies. In Kalker's variational approach, the so-called exact theory, the following assumptions are made:

- the bodies are formed of linearly elastic materials and are homogeneous,

- the contact area is essentially flat and small with respect to typical dimensions of the bodies' geometries,

- also, no sharp variations exist in the geometries of the bodies,

- inertial effects are small with respect to the contact stresses and may be ignored.

These assumptions allow for using the so-called half-space approach. The actual response of the bodies to the surface loading may be approximated with that of the elastic halfspace, which was presented in analytical form by Boussinesq and Cerruti $[23,24]$. With the half-space solution the contact problem is brought into surface-mechanical form:

$\mathbf{u}(\mathbf{x})=\int_{C} \mathbf{A}(\mathbf{x}, \mathbf{y}) \mathbf{p}(\mathbf{y}) d S$

In this form no reference is made to the stresses and/or displacements in the bodies' interiors.

A calculation in CONTACT is based on a rectangular "potential contact area" that is divided into rectangular elements of size $\delta x \times \delta y$. For each element $I$ it is determined whether it belongs to the adhesion (stick), slip or exterior areas $H, S$ and $E$. Equations (1)-(10) are discretised by inserting piecewise constant tractions $p_{I n}, \mathbf{p}_{I \tau}$. They are solved using active set algorithms NORM and TANG [7] and using a specific nonlinear Gauss-Seidel approach "ConvexGS" [25].
2.4 Hertzian solution to the normal problem, discretisation grid

The simplified theory is not reliable for the normal contact problem. Therefore in FASTSIM it is assumed that the normal problem has been solved and that the resulting contact area $C$ and traction bound $g$ are prescribed. In this work we confine ourselves to Hertzian geometries. Extension towards non-Hertzian problems is possible along the lines of $[12,26]$.

When the two bodies are geometrically and elastically similar ("quasi-identical"), the normal and tangential problems are uncoupled. Furthermore, if the undeformed distance $h(x, y)$ is quadratic in $x$ and $y$, the problem becomes "Hertzian". Its solution is analytically available due to Hertz [2]. The contact area is then elliptical, with semi-axes $a$ and $b$ in rolling and lateral directions, and the normal surface tractions are semi-ellipsoidal

$$
\begin{aligned}
p_{n}(x, y)= & \frac{3}{2 \pi} \frac{F_{n}}{a b}\left(1-\left(\frac{x}{a}\right)^{2}-\left(\frac{y}{b}\right)^{2}\right)^{1 / 2} \\
& (x, y) \in C .
\end{aligned}
$$

The elliptical Hertzian contact area is used in FASTSIM for establishing the grid. Different discretisation grids may be used. A simple option is to use $m x \times m y$ rectangular elements with size $\delta x \times \delta y$. In practice it is however more convenient to attune the grid to the Hertzian contact ellipse. As will be described below, there is no interaction in the model between tractions for different $y$-coordinates (lateral direction). A convenient choice is therefore to use different grid spacings in $x$-direction per slice, see Fig. 1:

$$
\begin{aligned}
& \delta y=\frac{2 b}{m y} \\
& y_{j}=-b+\left(j-\frac{1}{2}\right) \cdot \delta y, \quad j=1, \ldots, m y, \\
& x_{j}^{b n d}=a\left(1-\left(\frac{y_{j}}{b}\right)^{2}\right)^{1 / 2}, \\
& \delta x_{j}=\frac{2 x_{j}^{b n d}}{m x} \\
& x_{i, j}=x_{j}^{b n d}-\left(i-\frac{1}{2}\right) \cdot \delta x_{j}, i=1, \ldots, m x .
\end{aligned}
$$

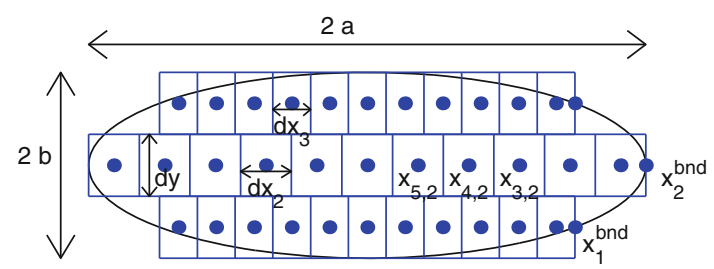

Fig. 1 Illustration of the grid used in the FASTSIM2 algorithm 
Using this "cell-centered" approach, the calculation of total force $\mathbf{F}$ (integral of tractions $\mathbf{p}$ over the contact area) is achieved easily using the mid-point rule, i.e. summing up the values $\mathbf{p}_{i, j}$ multiplied by the corresponding cell sizes $\delta x_{j} \times \delta y$. Further this choice simplifies the treatment of the edges of the contact area.

Substitution of (11) in (9) leads to a semi-ellipsoidal traction bound. This traction bound plays a vital role in FASTSIM. A drawback of the semi-ellipsoidal traction bound is that it prevents slip at the leading edge of the contact area. A parabolic traction bound is often used as an alternative:

$p_{n}(x, y)=\frac{2}{\pi} \frac{F_{n}}{a b}\left(1-\left(\frac{x}{a}\right)^{2}-\left(\frac{y}{b}\right)^{2}\right),(x, y) \in C$.

This modification is said to reproduce the subdivision of the contact area into adhesion and slip areas better, which is confirmed by the results presented in Section 5 .

\subsection{The simplified theory and corresponding FASTSIM algorithm}

The simplified theory is based on replacing the full constitutive behaviour (10) by the approximation

$\mathbf{u}(\mathbf{x})=L \mathbf{p}(\mathbf{x})$

That is, the surface particles $\mathbf{x}$ are assumed to move independently of each other and the response is linear in the surface traction $\mathbf{p}$, as if the bodies consist of a set of springs with flexibility parameter $L$. This is also called the Winkler (elastic) foundation model $[27,28]$. This approach is not reliable in the normal direction and the Hertzian approach must be used there. However, it does lead to quite acceptable results in the tangential direction using the FASTSIM algorithm [10].

The condition that the bodies' surfaces are free of traction in the exterior region, see (6), results in zero tangential traction at the leading edge. Using (14), this corresponds to zero tangential displacement $\mathbf{u}=\mathbf{0}$ at the leading edge. Starting at the leading edge, the slip equation (2) may be integrated along the trajectory of a particle through the contact area. Owing to the moving coordinate system, this trajectory is defined by the velocity vector $\mathbf{v}=[-V, 0]^{T}$. Due to orientation of $\mathbf{v}$ and the local nature of (14), there is no interaction in $y$-direction.

In the core of the FASTSIM algorithm, one finds the numerical integration of the slip equation (2). The presentation in [7] starts by discretising (2) in time:

$$
\begin{aligned}
\mathbf{s}(\mathbf{x}, t)= & \mathbf{w}(\mathbf{x}, t) \\
& +(\mathbf{u}(\mathbf{x}, t)-\mathbf{u}(\mathbf{x}-\delta t \mathbf{v}, t-\delta t)) / V \delta t .
\end{aligned}
$$

Setting $V \delta t=\delta x$, and assuming that $\mathbf{s}, \mathbf{w}$ and $\mathbf{u}$ do not depend on time, this yields:

$\mathbf{s}_{i, j}=\mathbf{w}_{i, j}+\left(\mathbf{u}_{i, j}-\mathbf{u}_{i-1, j}\right) / \delta x \leftrightarrow$

$\mathbf{u}_{i, j}=\mathbf{u}_{i-1, j}+\delta x \mathbf{s}_{i, j}-\delta x \mathbf{w}_{i, j}$.

This may be viewed as the "backward" or "implicit Euler" integration method for the differential equation $-\mathbf{u}^{\prime}=\mathbf{s}-\mathbf{w}$, which holds for a material particle along its characteristic line $\mathbf{x}=\mathbf{x}_{0}-\mathbf{v} t$. As will be shown in Section 5, this results in linear convergence of the total forces $\mathbf{F}$ when the step size $\delta x$ is refined. The presentation in [10] is different. It evaluates the rigid slip " $\mathbf{w}_{1 / 2}$ " at the intermediate point $x_{i-1 / 2, j}=$ $x_{i}-\delta x_{j} / 2$. Its notation "s $1 / 2$ " suggests that the slip is defined there too. However, careful consideration of the update-

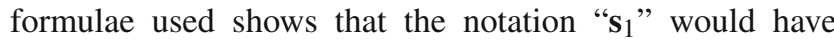
been more appropriate. This method, too, is only first-order accurate.

\subsection{Using three flexibilities in the FASTSIM algorithm}

One particular aspect of the FASTSIM algorithm concerns the choice of the flexibility parameter $L$. When the simplified theory is used as approximation to the theory of linear elasticity, there is no value directly available. This is solved by Kalker by relating the FASTSIM results to those of the linear theory for rolling contact $[7,29]$.

- If the creepages are "vanishingly small" then the total forces $F_{x}, F_{y}$ and the torsional moment $M_{z}$ are linear functions of the creepages $\xi, \eta, \phi$.

- Problems with a single creepage (either $\xi$ or $\eta$ or $\phi \neq 0$ ) may be solved analytically using the linear theory as well as with the simplified theory. This yields the flexbilities that give identical results in both theories:

$L_{\xi}^{\prime}=\frac{8 a}{3 C_{11} G}, \quad L_{\eta}^{\prime}=\frac{8 a}{3 C_{22} G}, \quad L_{\phi}^{\prime}=\frac{\pi a \sqrt{a / b}}{4 C_{23} G}$

- In problems with mixed creepages, a linear combination of the separate solutions may be used. This linear combination may be used as "best guess" in problems with larger creepages, where the assumption of linearity no longer holds.

As far as the tractions $\mathbf{p}$ are concerned, the superimposed problems with flexibilities according to (17) and rigid slip (3) are mathematically equivalent to a problem with "effective rigid slip"

$\tilde{\mathbf{w}}=\left[\frac{\xi}{L_{\xi}^{\prime}}-\frac{y \phi}{L_{\phi}^{\prime}}, \frac{\eta}{L_{\eta}^{\prime}}+\frac{x \phi}{L_{\phi}^{\prime}}\right]^{T}$ 
and with flexibility $\tilde{L}=1$. This is however not true for the displacements $\mathbf{u}$. In the absence of spin creepage, (18) amounts to an anisotropic variant of (14). One effect of this is that the displacements $\mathbf{u}$ and tractions $\mathbf{p}$ are no longer co-linear. When spin is present, (18) can no longer be interpreted as constitutive model in the form of (14).

The alternative to using (17), (18) is to use a weighted mean of the flexibilities:

$L=\left(|\xi| L_{\xi}^{\prime}+|\eta| L_{\eta}^{\prime}+\sqrt{a b}|\phi| L_{\phi}^{\prime}\right) / \sqrt{\xi^{2}+\eta^{2}+a b \phi^{2}}$.

This reduces the agreement of FASTSIM to the exact theory. Therefore we use the three flexibilities of (17) and (18) in our experiments. In the derivation of the equations we ignore the difference in direction of $\mathbf{u}$ and $\mathbf{p}$. In the remainder of this paper we therefore concentrate on the approach with a scalar flexibility $L$.

\section{Analysis of a 1D model problem}

\subsection{A model problem}

In order to get insight in the behaviour of the contact problem (2)-(9), (14), we consider a 1D model problem first.

$$
\begin{aligned}
& \frac{d u(x)}{d x}-s(x)=-w(x), \quad x \in[0,1], \\
& u(0)=0, \\
& |u(x)| \leq u_{\max }(x),
\end{aligned}
$$

adhesion area : $s=0, \quad|u| \leq u_{\max }$,

slip area : $s \neq 0, \quad|u|=u_{\max }, \quad s \cdot u \leq 0$.

Here $u(x), s(x)$ and $w(x)$ represent the $x$-component (longitudinal) of the displacement difference $\mathbf{u}$, slip $\mathbf{s}$ and rigid slip $\mathbf{w}$ respectively. The coordinate and variables are scaled such that the contact patch has unit length and such that the resulting traction bound, expressed in $u$ and denoted by $u_{\max }$, has simple coefficients and is a nonnegative function. Moreover, the coordinate direction is reversed in order to have a grid going from low $x$ to high $x$.

Below, we consider some specific examples and present analytical solutions followed by a discussion of the numerical treatment.

Although this shows that it is possible to solve the problem (20) analytically, it must be realized that the analytical approach cannot be extended easily for $2 \mathrm{D}$ problems or for problems with more general rigid slip and/or traction bounds. However, the analytical approach clearly reveals the most important mechanisms and this helps to sharpen the mind in the process of developing numerical procedures.
3.2 Analytical treatment of the 1D problem

Case 1: parabolic traction bound and small spin First we consider the system (20) with

$u_{\max }=4 x(1-x), \quad w(x)=-0.1-12 x$

This concerns a parabolic traction bound and a relatively small amount of spin.

Around $x=0$, it is seen that $|w|<\left|d u_{\max } / d x\right|$ and, as a consequence, the analytical solution starts with a region of adhesion $(s=0)$. Therefore, we start with integrating $-w(x)$. At $x=0.39$, the solution hits $+u_{\max }$. At $x=0.39$, it holds that $d u_{\max } / d x$ is smaller than $-w$, and this means that, in order to satisfy $|u| \leq u_{\max }$, the solution continues with a region of $\operatorname{slip}(s<0)$. To summarize the solution reads

$\begin{cases}u(x)=0.1 x+6 x^{2}, s(x)=0, & x \leq 0.39 \\ u(x)=u_{\text {max }}, s(x)=3.9-20 x, & x>0.39\end{cases}$

It is important to note that the slip $s(x)$ is discontinuous at the transition from adhesion to slip, and that the displacement $u(x)$ is continuous with a discontinuous derivative.

Case 2: parabolic traction bound and large spin As a second example we consider the system (20) with

$u_{\max }=4 x(1-x), \quad w(x)=-6+14 x$

This concerns a parabolic traction bound and a large amount of spin.

Around $x=0$, it is seen that $|w|>\left|d u_{\max } / d x\right|$ and, as a consequence, the analytical solution starts with a region of slip. At $x=0$, it holds that $w<0$ and this means that, in order to satisfy $s \cdot u \leq 0$, the solution starts at the maximum $+u_{\max }$. For $u>0$, transition to adhesion will take place as soon as $-w$ drops below $+d u_{\max } / d x$, which is at $x=1 / 3$. From that point we integrate $-w(x)$ to obtain

$u(x)=8 / 9+\int_{1 / 3}^{x}-w\left(x^{\prime}\right) d x^{\prime}=-1 / 3+6 x-7 x^{2}$.

At the transition from slip to adhesion, the slip $s(x)$ is continuous $(s=0)$. At $x \approx 0.8744$, the solution hits $-u_{\max }$. At $x \approx 0.8744$, it holds that $-w<-d u_{\max } / d x$ and this means that the solution continues with a region of $\operatorname{slip}(s>0)$. To summarize the solution reads

$$
\left\{\begin{array}{l}
u(x)=u_{\max }, s(x)=6 x-2, \quad x<1 / 3 \\
u(x)=-7 x^{2}+6 x-1 / 3, s(x)=0, \quad 1 / 3 \leq x \leq 0.8744 \\
u(x)=-u_{\max }, s(x)=22 x-10, \quad 0.8744<x
\end{array}\right.
$$

As before, the slip is discontinuous at the transition from adhesion to slip. 
Case 3: ellipsoidal traction bound Thirdly, we consider the situation with an ellipsoidal traction bound

$u_{\max }=\sqrt{4 x(1-x)}, \quad w(x)=-6+14 x$

In this case the derivative of $u_{\max }$ at $x=0$ is infinite, which prevents slip at the leading edge. The adhesion solution is again determined by integrating $-w(x)$. As soon as the solution hits $+u_{\max }$, which occurs at $x \approx 0.1355$, a transition to slip takes place $\left(u>0, d u_{\max } / d x<-w\right)$. After this $u=$ $u_{\text {max }}$. A transition to adhesion ( $-w$ drops below $d u_{\max } / d x$ ) is found at $x \approx 0.3992$. Finally, there is another transition to slip, where $u(x)$ reaches $-u_{\max }$, that is, at $x \approx 0.9011$. To summarize the solution reads

$$
\left\{\begin{array}{l}
u(x)=6 x-7 x^{2}, \quad s(x)=0, \quad x \leq 0.1355, \\
u(x)=u_{\max }, \quad s(x)<0, \quad 0.1355<x<0.3992, \\
u(x)=-0.3203+6 x-7 x^{2}, \quad s(x)=0, \\
\quad 0.3992 \leq x \leq 0.9011, \\
u(x)=-u_{\max }, \quad s(x)>0, \quad 0.9011<x .
\end{array}\right.
$$

To establish the coordinates at which the transitions take place, fourth-order polynomial equations must be solved.

\subsection{Numerical treatment of the 1D problem}

The use of standard numerical integration techniques (see e.g. [30]) requires that the problem is formulated in the appropriate form. In our case this form is

$\frac{d u}{d x}=f(x, u), \quad 0<x \leq 1, \quad u(0)=0$

In this standard form auxiliary conditions such as given by the constraints (20c)-(20e) are incorporated implicitly. This requires a right-hand side function $f$ that depends on $w$ and $u_{\max }$ in a complicated way, with switches depending on whether $u$ is below or at the traction bound $u_{\text {max }}$. This allows standard solvers, such as Matlab's ODE solvers, to be used. However, an experiment in this direction shows that this does not yield a satisfactory result. The standard solvers tried (RK4, ODE45) either use many small integration steps near the discontinuities, or yield a significant overshoot over the traction bound.

Instead, in this section we consider specific numerical integration schemes for problem (20). In these specific schemes we do not transform the problem to a standard form, but use the constraints explicitly to develop an appropriate algorithm.

The first strategy employed, concerns the original FASTSIM algorithm. In the present context, (16) becomes

$u_{i}=u_{i-1}+\delta x s_{i}-\delta x w_{i}$,

i.e. the backward Euler integration rule for (20a) [30]. For the remainder of the paper, the following is important. Update formulae, like (29), are in the core. We often use the formula to compute first a trial solution at $x=x_{i}$. For this, we assume that this point is in the adhesion region and replace $s_{i}=s_{i}^{a d h}$ by 0 in the update formula. The corresponding trial solution is called the adhesion solution and carries the superscript $a d h$. With this convention FASTSIM reads:

0 . Choose the number of points $m x$, set $\delta x=1 / m x$ and $x_{i}=i \cdot \delta x$, for $i=0, \ldots, m x$.

1. Start with $u_{0}=0$ and step from left to right through the gridpoints.

2. For each new point $x_{i}$, assume that it is in the adhesion region and compute the trial adhesion solution $u_{i}^{a d h}=$ $u_{i-1}-\delta x w_{i}, s_{i}^{a d h}=0$.

3. If $\left|u_{i}^{a d h}\right| \leq u_{\max , i}$ then set $u_{i}=u_{i}^{a d h}, s_{i}=0$.

4. Else, set

$$
\begin{aligned}
u_{i} & =\operatorname{sign}\left(u_{i}^{a d h}\right) u_{\max , i}, \\
s_{i} & =\left(u_{i}-u_{i-1}\right) / \delta x+w_{i} .
\end{aligned}
$$

Equation (30a) is an extension of the integration scheme to incorporate the auxiliary conditions (20c-20e). Equation (30b) corresponds to a one-sided numerical differentiation of the function $u(x)$.

As being based on (29), the approximations are only firstorder accurate. An alternative is provided by the so-called $\theta$-method, which is our second strategy:

$$
\begin{aligned}
& \frac{u_{i}-u_{i-1}}{\delta x}-\left(\theta s_{i}+(1-\theta) s_{i-1}\right) \\
& =-\left(\theta w_{i}+(1-\theta) w_{i-1}\right) .
\end{aligned}
$$

For $\theta=1 / 2$, this yields the Crank-Nicholson method or trapezoidal rule [30]. For $\theta=1$, this coincides with the backward Euler method. This method may be employed using the following steps:

1'. Start with $u_{0}=0$. If $\left|w_{0}\right| \leq u_{\text {max }}^{\prime}(0)$ set $s_{0}=0$, else make an estimate for $s_{0}$.

2'. For each new point $x_{i}$, compute the trial adhesion solution $u_{i}^{a d h}$ from (31) with $s_{i}^{a d h}=0$.

3'. If $\left|u_{i}^{a d h}\right| \leq u_{\max , i}$ then $u_{i}=u_{i}^{a d h}, s_{i}=0$,

4'. Else, set $u_{i}=\operatorname{sign}\left(u_{i}^{a d h}\right) u_{\max , i}$ and use (31) to determine $s_{i}$.

The introduction of $s_{0}$ in step 1' is needed because of the occurrence of $s_{i-1}$ in equation (31). If $\left|w_{0}\right|>u_{\text {max }}^{\prime}(0)$ then there is slip at the leading edge, e.g. see case 2 in Section 3.2. It is then needed to have access to $u_{\text {max }}^{\prime}(0)$ (or a numerical approximation). The slip $s_{0}$ may be estimated as

$s_{0}=w_{0}+\operatorname{sign}\left(-w_{0}\right) u_{\text {max }}^{\prime}(0)$.

It is possible to recast step 4' as

$s_{i}=\frac{u_{i}-u_{i}^{a d h}}{\theta \delta x}$. 

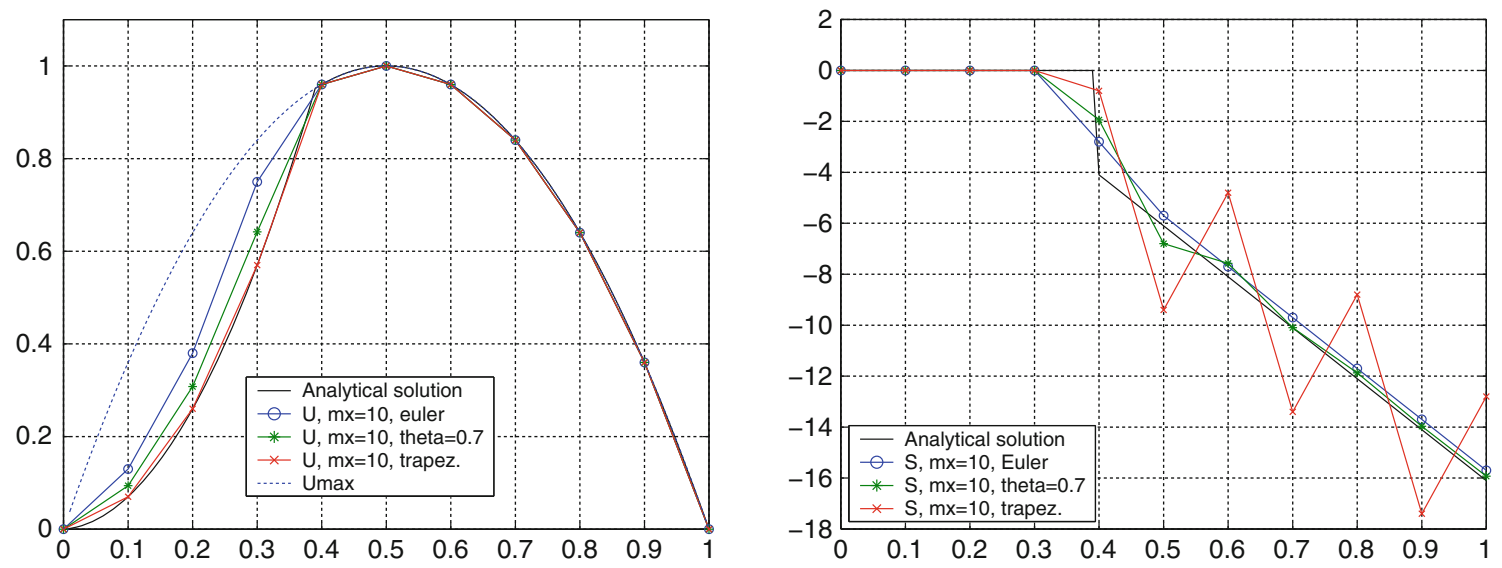

Fig. 2 Results for the backward Euler and $\theta$-methods for case 1 on a grid of 10 points. Left solution $u(x)$ for $\theta=1.0,0.7$ and 0.5 . Right corresponding $\operatorname{slip} s(x)$

Written in this manner, this step may be referred to as to compute the slip needed to arrive back from $u_{i}^{a d h}$ at the traction bound.

Results for case 1 of Section 3.2 are shown in Fig. 2. It can be observed that the Euler method introduces large errors in $u(x)$ and an offset error in $s(x)$. The trapezoidal rule reproduces the exact solution for $u(x)$, which is expected because $w(x)$ is linear. However, it also shows a significant oscillation in $s(x)$. This is typical for the trapezoidal rule. A partial solution is to increase $\theta$ a bit, which damps the oscillation. This, however, reduces the accuracy of the results.

Improvements of these results can be achieved by paying special attention to internal boundaries, i.e. the points at which transition takes place. This is our third strategy. It requires the location $x_{i n t}$ of the internal boundaries and employs fractional integration steps.

3"(a) If $\left|u_{i}^{a d h}\right| \leq u_{\max , i}$ and $s_{i-1}=0$ (adhesion, previous element in adhesion too) then $u_{i}=u_{i}^{a d h}, s_{i}=0$, as in step 3' above,

3"(b) Else, if $\left|u_{i}^{a d h}\right| \leq u_{\max , i}$ (transition from slip to adhesion), a trial slip solution is used. $u_{i}^{s l p}$ is set to $\pm u_{\max , i}$ and $s_{i}^{s l p}$ is computed from (31). Note that $s_{i-1}$ and $s_{i}^{s l p}$ have opposite signs, and that the associated trial slip solution $s^{s l p}(x)$ is continuous and has a zero value at $x_{i n t}$. A linear approximation of $s^{s l p}(x)$ is used to estimate the position $x_{i n t}$. This yields the exact $x_{i n t}$ for a parabolic traction bound, and is sufficiently accurate for an ellipsoidal traction bound too. Finally, an accurate approximation of $u_{i}$ is obtained by setting $u_{\text {int }}=u_{\max }\left(x_{i n t}\right), s_{i n t}=0$ and applying (31) on the interval from $x_{i n t}$ to $x_{i}$.

4"(a) Else, if $s_{i-1} \neq 0$ (slip, previous element in slip area too) set $u_{i}=\operatorname{sign}\left(u_{i}^{a d h}\right) u_{\max , i}$ and use (31) to determine $s_{i}$, as in step 4' above, 4"(b) Else (transition from adhesion to slip), the location $x_{i n t}$ of the internal boundary is determined by estimating where the trial adhesion solution crosses the traction bound. An intermediate point $x_{i-1 / 2}$ is introduced and the adhesion solution in this point is computed using (31) with step size $\delta x / 2$. A quadratic fit is done on the function $f=\left|u^{a d h}\right|-u_{\max }$ using the values of $f$ at the points $x_{i-1}, x_{i-1 / 2}, x_{i}$. Next, we set $u_{\text {int }}= \pm u_{\max }\left(x_{i n t}\right), u_{i}= \pm u_{\max }\left(x_{i}\right)$. The slip after its jump at the internal boundary may be evaluated similar to (32), i.e.

$$
s_{i n t}=w_{i n t}+\operatorname{sign}\left(u_{i n t}\right) u_{\max }^{\prime}\left(x_{i n t}\right) .
$$

Finally, an accurate approximation of $s_{i}$ may be established from a fractional integration step, i.e. applying (31) on the interval from $x_{i n t}$ to $x_{i}$.

With these extensions, the analytical solution is recovered perfectly for $\theta=0.5$ when a parabolic traction bound is used.

\section{The FASTSIM2 approach}

The numerical approach, outlined above for the 1D test problem, has been used to extend the FASTSIM approach in order to make it second-order accurate. This new approach is called FASTSIM2 and is detailed in this paragraph.

In FASTSIM2, the slices in lateral direction can be treated independently. Therefore, for the ease of presentation, the index $j$ is ignored. We just show how one slice can be treated and, by repetition, all slices can be done.

As in the previous section, the trial solution $\mathbf{u}_{i}^{a d h}$ is computed with corresponding traction $\mathbf{p}_{i}^{a d h}=L^{-1} \mathbf{u}_{i}^{a d h}$. Next, it is checked whether the traction bound is exceeded $\left(\left\|\mathbf{p}_{i}^{a d h}\right\|>\right.$ 
$g_{i}$ ). If so, the slip solution $\mathbf{u}_{i}^{s l p}, \mathbf{p}_{i}^{s l p}$ is inserted. Because both a magnitude (equal to $g_{i}$ ) and a direction are involved, details are a little bit more complicated than in the 1D case. Using a single flexibility $L$, the vectors $\mathbf{u}_{i}^{s l p}, \mathbf{u}_{i}^{a d h}, \mathbf{p}_{i}^{s l p}$ and $\mathbf{p}_{i}^{a d h}$ are all co-linear, which is used to compute the slip solution. If the flexibilities are not equal, then we keep on using this, assuming that a reasonable approximation results.

It has been found in the previous section that the choice $\theta=0.5$, leading to the trapezoidal rule, is the most attractive. Therefore, this value is adapted in the present section. This means that in the present context (31) becomes

$\mathbf{u}_{i}=\mathbf{u}_{i-1}+\frac{\delta x}{2}\left(\mathbf{s}_{i}+\mathbf{s}_{i-1}\right)-\frac{\delta x}{2}\left(\mathbf{w}_{i}+\mathbf{w}_{i-1}\right)$,

$\mathbf{p}_{i}=L^{-1} \mathbf{u}_{i}$.

To finalize the FASTSIM2 algorithm becomes:

0. Choose the number of points $m x$, set $\delta x, x_{0}=x_{b n d}$ and $x_{i}$ as described in Section 2.4.

1. Start with $\mathbf{u}_{0}=\mathbf{p}_{0}=\mathbf{0}$, estimate $\mathbf{s}_{0}$ when required, and step from right to left through the grid points. Note that the first step $i=1$ employs grid size $\delta x / 2$.

2. For each new point $x_{i}$, compute the trial adhesion solution $\mathbf{u}_{i}^{\text {adh }}$ from (35) (replace $\mathbf{s}_{i}$ by $\mathbf{s}_{i}^{\text {adh }}=\mathbf{0}$ ).

3. If $\left\|\mathbf{p}_{i}^{a d h}\right\| \leq g_{i}$, then adhesion occurs and $\mathbf{s}_{i}=\mathbf{0}$. Two situations are possible.

(a) In case $\mathbf{s}_{i-1}=\mathbf{0}$, the step is fully in the adhesion region and $\mathbf{u}_{i}=\mathbf{u}_{i}^{a d h}, \mathbf{p}_{i}=\mathbf{p}_{i}^{a d h}$.

(b) In case $\mathbf{s}_{i-1} \neq \mathbf{0}$, an internal boundary with a transition from slip to adhesion is present. Its location $x_{i n t}$ is determined as a zero of the trial slip solution, see the previous section and equations (37) and (38) below. In this case the trial slip solution is vectorial. To determine $x_{i n t}$, it is not a very good idea to search for a zero of $\left\|\mathbf{s}^{s l p}\right\|$. What we did, is to determine the largest component of the slip vector $\mathbf{s}_{i-1}$ and to focus on this component. During the step, this component crosses zero and $x_{i n t}$ has been determined from this.

Knowing that $x_{i n t}$ is in the adhesion region, it holds that $\mathbf{s}_{\text {int }}=\mathbf{0}$. Next, the displacement $\mathbf{u}_{i}$ follows from applying (35) twice, on the intervals $\left[x_{i n t}, x_{i-1}\right]$ and $\left[x_{i}, x_{i n t}\right]$. In the first $\mathbf{u}_{i n t}$ is computed and in the second $\mathbf{u}_{i}$ :

$$
\begin{aligned}
& \frac{\mathbf{u}_{i n t}-\mathbf{u}_{i-1}}{\left|x_{i n t}-x_{i-1}\right|}=\frac{1}{2} \mathbf{s}_{i-1}-\frac{1}{2}\left(\mathbf{w}_{i n t}+\mathbf{w}_{i-1}\right), \\
& \frac{\mathbf{u}_{i}-\mathbf{u}_{i n t}}{\left|x_{i}-x_{i n t}\right|}=-\frac{1}{2}\left(\mathbf{w}_{i}+\mathbf{w}_{i n t}\right) .
\end{aligned}
$$

4. If $\left\|\mathbf{p}_{i}^{a d h}\right\|>g_{i}$, then slip takes place and the tractions are scaled to be on the traction bound. This leads to

$\mathbf{p}_{i}=g_{i} \frac{\mathbf{p}_{i}^{a d h}}{\left\|\mathbf{p}_{i}^{a d h}\right\|}, \quad \mathbf{u}_{i}=L \mathbf{p}_{i}$.

For determining the slip, two situations must be distinguished:

(a) In case $\mathbf{s}_{i-1} \neq \mathbf{0}$, the step is fully in the slip region and (35) is used to determine $\mathbf{s}_{i}$. This is conveniently achieved by subtracting (35) for $\mathbf{u}_{i}^{a d h}$ from (35) for $\mathbf{u}_{i}$, which yields

$\mathbf{s}_{i}=2 \frac{\mathbf{u}_{i}-\mathbf{u}_{i}^{a d h}}{\delta x}$,

which corresponds to equation (33) for the 1D case.

(b) In case $\mathbf{s}_{i-1}=\mathbf{0}$, the step involves an internal boundary with a transition from adhesion to slip. Its location $x_{i n t}$ is determined as a zero of a quadratic approximation of $f=\left\|\mathbf{p}^{a d h}\right\|-\mu p_{n}$, see the previous section for all details concerning the root finding process. It appears to be difficult to estimate the slip $\mathbf{s}_{i n t}$ at the internal boundary, which is therefore approximated by $\mathbf{s}_{i}$. Next, the slip $\mathbf{s}_{i}$ follows from applying two fractional integration steps. In the first $\mathbf{u}_{\text {int }}$ is computed and in the second $\mathbf{s}_{i}$ :

$$
\begin{aligned}
& \frac{\mathbf{u}_{i n t}-\mathbf{u}_{i-1}}{\left|x_{i n t}-x_{i-1}\right|}=-\frac{1}{2}\left(\mathbf{w}_{i n t}+\mathbf{w}_{i-1}\right), \\
& \frac{\mathbf{u}_{i}-\mathbf{u}_{i n t}}{\left|x_{i}-x_{i n t}\right|}=\mathbf{s}_{i}-\frac{1}{2}\left(\mathbf{w}_{i}+\mathbf{w}_{i n t}\right) .
\end{aligned}
$$

It might be that there is slip at the leading edge. In order to cope with this situation, the slip $\mathbf{s}_{0}$ is estimated in step 1 . The condition for slip at the leading edge becomes $\|\mathbf{w}(0) / L\|>$ $\partial g / \partial x$, and the slip is given by

$\mathbf{s}_{0}=\left(\left\|\mathbf{w}_{0}\right\|-L g_{0}\right) \frac{\mathbf{w}_{0}}{\left\|\mathbf{w}_{0}\right\|}$

This follows from the boundary condition $\mathbf{u}_{0}=\mathbf{0}$, which makes $d \mathbf{u} / d x$ co-linear with $\mathbf{u}$, such that $\mathbf{s}$ is co-linear with $\mathbf{w}$ near the leading edge.

\section{Discretisation accuracy of FASTSIM and FASTSIM2 algorithms}

For assessing the discretisation accuracy we use the methodology presented in [21]. This strategy consists of defining an appropriate test-set, choosing the reference results, and then presenting the statistics of the differences. In the present evaluation the same test-set is used as in [21]. It comprises 3,220 combinations of contact patch ellipticities and creepage 
values, which cover the range of parameter values that occur for realistic vehicles and tracks in VSD simulations.

The main parameters of interest to VSD packages are not the tractions $\mathbf{p}$, but the resulting total forces $F_{\tau}, \tau=x, y$. These are obtained by integrating the components $p_{\tau}$ of $\mathbf{p}$ over the contact area. The resulting values are scaled by $\mu F_{n}$ in order to obtain relative total forces, which lie in the range $[-1,1]$. Note that the accuracy of $\mathbf{p}$ (and $\mathbf{s}$ ) may be of interest as well, for instance for the computation of wear.

Figure 3 shows the accuracy of the FASTSIM2 results when compared to the CONTACT results, when using a fine discretisation grid of $640 \times 640$ points. The figure displays the distribution of the errors $\left|F_{\tau}^{\text {fastsim } 2}-F_{\tau}^{\text {contact }}\right|$. For instance it can be seen that the difference in $F_{x}$ between FASTSIM2 and CONTACT is $70 \%$ of the time less than 0.02 and $90 \%$ of the time less than 0.04. A few statistics of the differences are presented in Table 1. These results correspond to those of the FASTSIM algorithm presented in [21]. When finer and finer discretisation grids are used, both methods converge to the same result.

The figure and table present a comparison of the use of one or three flexibilities and of the parabolical and semiellipsoidal traction bounds as well. This shows that, as far as the total forces are concerned, the use of three flexibilities clearly improves the accuracy over using a single flexibility.
When using three flexibilities, the parabolical traction bound yields results slightly closer to those of CONTACT than when the semi-ellipsoidal traction bound is used. Since the parabolical bound is expected to yield more realistic adhesion and slip areas as well, and since it can be dealt with more easily in the FASTSIM2 algorithm, it is advised against using the semi-ellipsoidal traction bound.

Next we turn to the discretisation errors in the old and new algorithms. Figure 4 and Table 2 show the grid convergence of the results for the FASTSIM and FASTSIM2 algorithms. Each line in the graphs compares the total forces $F_{y}$ obtained for one grid, for instance using $m x=m y=20$, to the values computed with FASTSIM 2 on a very fine grid. This then concerns the errors due to discretisation effects alone. In the left figure, for the original FASTSIM algorithm, the errors are halved each time the grid is refined. In the figure on the right, for FASTSIM2, the errors are reduced by a factor of three to four each time the grid is refined.

A closer study has revealed that the errors in the original FASTSIM algorithm are linear in the grid size $\delta x$ and quadratic in the grid size $\delta y$. This is seen for instance when a graph comparable to Fig. 4 is made, where $m x$ is held constant at, say, 160 and $m y$ is varied. The errors related to the $y$-direction are much smaller than those related to $\delta x$. Consequently the accuracy is not harmed if the number of elements
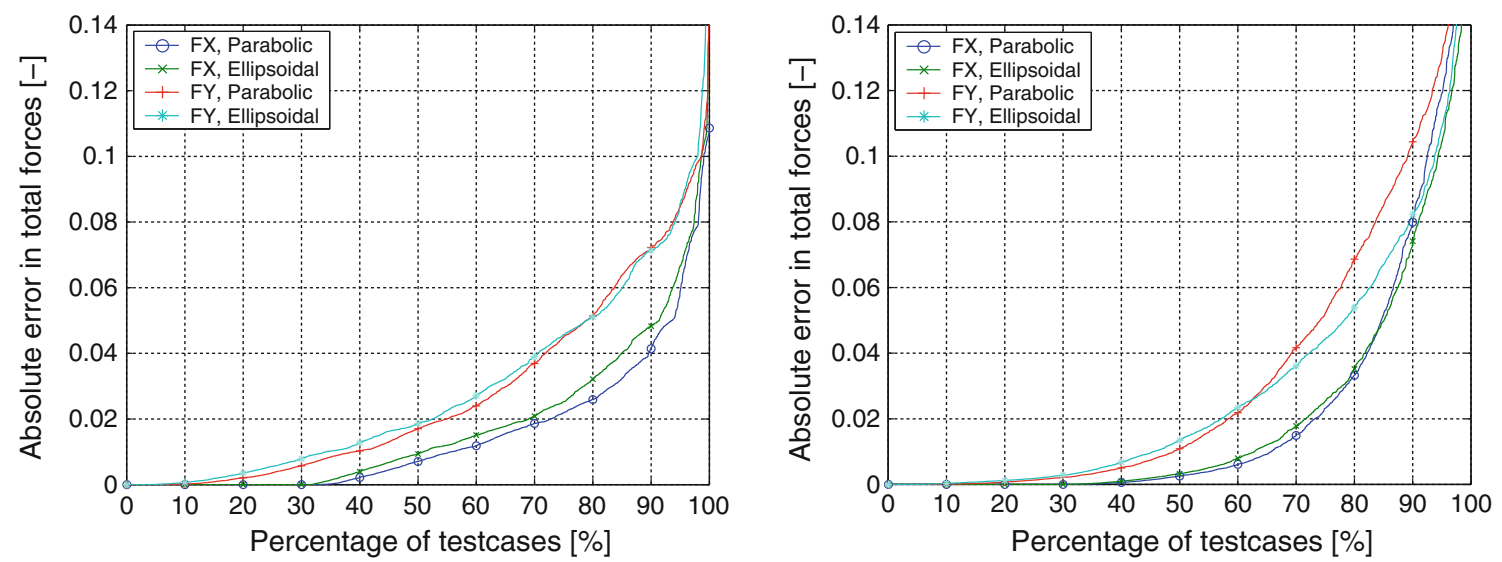

Fig. 3 Accuracy of the relative total forces $F_{x}$ and $F_{y}$ computed with the FASTSIM2 algorithm on a fine grid w.r.t. CONTACT results. Left using three flexibilities (17), (18); right using scalar flexibility (19)

Table 1 Statistics of the errors in total forces $F_{x}$ and $F_{y}$ computed by FASTSIM 2 using a $640 \times 640$ grid compared to those computed by CONTACT

\begin{tabular}{|c|c|c|c|c|c|c|}
\hline & \multicolumn{3}{|c|}{ Errors in $F_{x}$} & \multicolumn{3}{|c|}{ Errors in $F_{y}$} \\
\hline & $80 \%$ & $95 \%$ & $\mathrm{rms}$ & $80 \%$ & $95 \%$ & rms \\
\hline \multicolumn{7}{|l|}{ Using three flexibilities (17), (18): } \\
\hline Parabolic tract.bound & 0.026 & 0.059 & 0.026 & 0.051 & 0.084 & 0.039 \\
\hline Semi-ellipsoidal tract.bound & 0.032 & 0.067 & 0.029 & 0.051 & 0.085 & 0.041 \\
\hline \multicolumn{7}{|l|}{ Using scalar flexibility (19): } \\
\hline Parabolic tract.bound, $L_{1}$ & 0.033 & 0.119 & 0.046 & 0.069 & 0.130 & 0.056 \\
\hline Semi-ellipsoidal tract.bound, $L_{1}$ & 0.035 & 0.104 & 0.041 & 0.054 & 0.109 & 0.049 \\
\hline
\end{tabular}



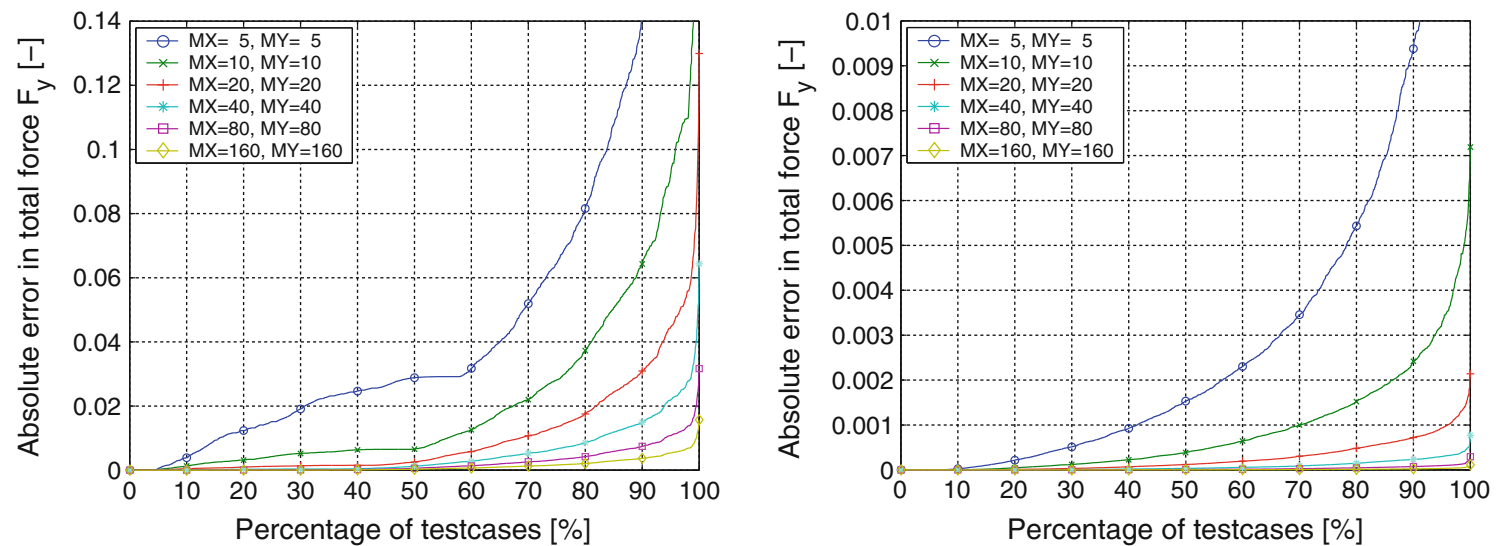

Fig. 4 Discretisation error in lateral force $F_{y}$ when using different grids. Left original FASTSIM algorithm; right FASTSIM2 algorithm. Note the difference in scales

Table 2 Statistics of the errors in total forces $F_{x}$ and $F_{y}$ computed by FASTSIM and FASTSIM2 compared to those of FASTSIM2 on a fine grid

\begin{tabular}{|c|c|c|c|c|c|c|}
\hline & \multicolumn{3}{|c|}{ Errors for FASTSIM } & \multicolumn{3}{|c|}{ Errors for FASTSIM2 } \\
\hline & $80 \%$ & $95 \%$ & $\mathrm{rms}$ & $80 \%$ & $95 \%$ & $\mathrm{rms}$ \\
\hline \multicolumn{7}{|l|}{ Errors in $F_{x}$ : } \\
\hline$m x=m y=5$ & 0.037 & 0.082 & 0.0368 & 0.004 & 0.011 & 0.00538 \\
\hline$m x=m y=10$ & 0.015 & 0.038 & 0.0162 & 0.001 & 0.003 & 0.00107 \\
\hline$m x=m y=20$ & 0.007 & 0.019 & 0.0077 & 0.000 & 0.001 & 0.00027 \\
\hline$m x=m y=40$ & 0.003 & 0.009 & 0.0037 & 0.000 & 0.000 & 0.00008 \\
\hline$m x=m y=80$ & 0.002 & 0.004 & 0.0017 & 0.000 & 0.000 & 0.00003 \\
\hline$m x=m y=160$ & 0.001 & 0.002 & 0.0007 & 0.000 & 0.000 & 0.00001 \\
\hline \multicolumn{7}{|l|}{ Errors in $F_{y}$ : } \\
\hline$m x=m y=5$ & 0.081 & 0.190 & 0.0814 & 0.005 & 0.013 & 0.02020 \\
\hline$m x=m y=10$ & 0.037 & 0.091 & 0.0379 & 0.002 & 0.003 & 0.00143 \\
\hline$m x=m y=20$ & 0.017 & 0.043 & 0.0180 & 0.000 & 0.001 & 0.00041 \\
\hline$m x=m y=40$ & 0.008 & 0.021 & 0.0086 & 0.000 & 0.000 & 0.00013 \\
\hline$m x=m y=80$ & 0.004 & 0.009 & 0.0040 & 0.000 & 0.000 & 0.00004 \\
\hline$m x=m y=160$ & 0.002 & 0.004 & 0.0017 & 0.000 & 0.000 & 0.00001 \\
\hline
\end{tabular}

$m y$ is reduced. Whereas Table 2 is concerned, about the same accuracy is obtained for the original FASTSIM algorithm if no more than 20 elements in $y$-direction are used.

Figure 5 shows the final results in a different way. This figure contains the RMS discretisation errors for the FASTSIM and FASTSIM2 algorithms. The triangles show the slopes associated to linear and quadratic convergence. This shows that quadratic convergence is not reached entirely. The difference is due to the use of three flexibilities. When just a single flexibility the RMS error is reduced by a factor 4 with each grid refinement.

These results demonstrate the superior behavior of FASTSIM2 with respect to discretisation accuracy. To quantify the benefit, a possible criterion for selecting $m x$ and $m y$ is used. This criterion is that the errors due to discretisation should be significantly (ten times) smaller than the error in the sim- plified theory itself, as shown in Table 1, illustrated by the dashed line in Fig. 5. Using this criterion, we would choose a $80 \times 20$ grid for the FASTSIM algorithm and $10 \times 10$ (or less) points when using FASTSIM2. This yields a 16 times reduction of the amount of computations to be performed.

\section{Conclusions and discussion}

In this paper we have considered the steady state rolling contact problem with dry friction and its approximation using the simplified theory. We have shown that the well-known FASTSIM algorithm can be seen as applying the backward Euler integration rule for the discretisation of the slip of material points. This approach is only first-order accurate in the step size $\delta x$ that is used and therefore requires fine grids. 

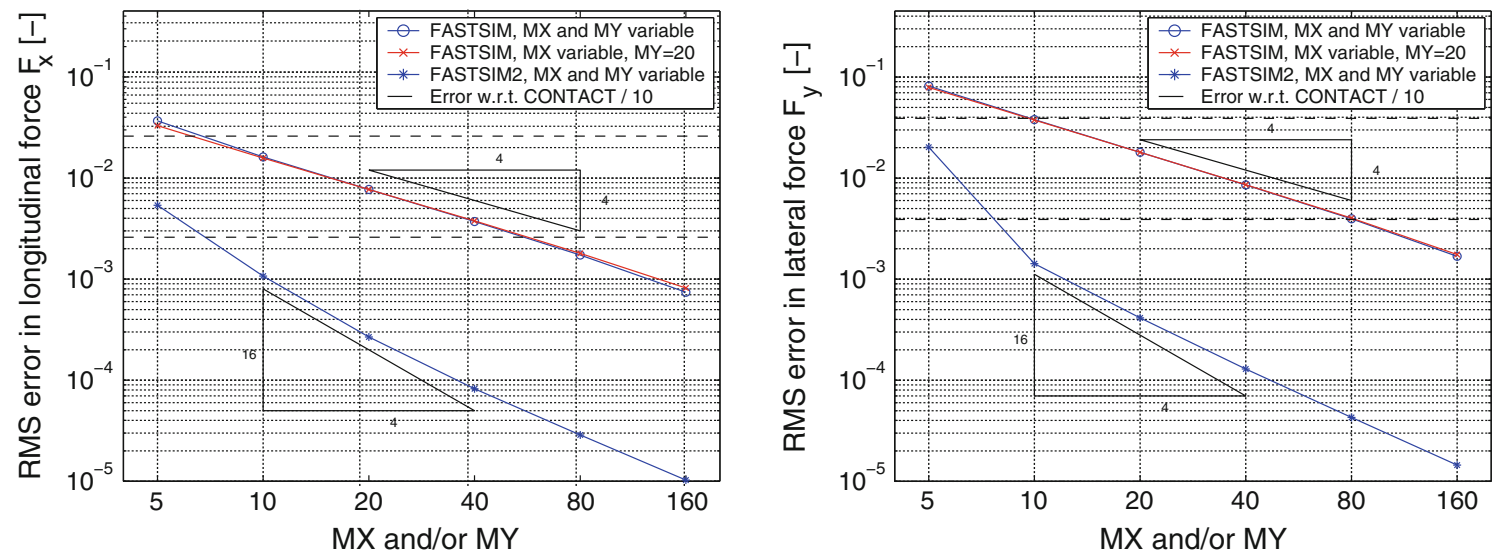

Fig. 5 Rms values of discretisation errors in total forces $F_{x}(l e f t)$ and $F_{y}(r i g h t)$ in FASTSIM and FASTSIM2

To improve, we have formulated a second-order successor FASTSIM2.

A one-dimensional model problem was put forward and used to back-up the development of a new discretisation approach. We were able to analyse the model problem analytically, revealing the different mechanisms at work in the adhesion and slip regions and the behavior of solutions at the internal boundaries. It was shown that the second-order accurate trapezoidal rule may be applied, improves the results, but also comes with spurious oscillations in the slip if internal boundaries are present. These oscillations could be suppressed by implementing an interface tracking approach, and by making precise estimates of the slip at the leading edge and at the internal boundaries.

The approach developed for the 1D problem has been extended to 2D in order to create our new FASTSIM2 algorithm. It was shown that this new algorithm has second-order convergence in the grid sizes $\delta x$ and $\delta y$, and that it needs only a grid of $10 \times 10$ points to get a sufficiently accurate result. In the original FASTSIM algorithm, grids that contain at least 16 times as many points are needed for a comparable accuracy.

In this paper, we have confined ourselves to Hertzian geometries and a Coulomb friction law. The main reason for this is to ease the exposition of the new approach, and for assessing the discretisation accuracy. The extensions that were made previously to the FASTSIM algorithm, can equally well be made to the FASTSIM2 algorithm.

The main point of the new strategy is that it recognizes the importance of the equation that describes the slip. The contact problem was previously viewed as a problem of linear elasticity, i.e. a problem with elliptical properties. It was more or less ignored that the slip equation describes a transport phenomenon and, as such, introduces hyperbolic properties. It seems to be possible to use this idea to improve the discretisation accuracy of CONTACT as well. CONTACT has also been found to be only first-order accurate [31]. Moreover, where the computing time for FASTSIM is linear in the number of elements $m x \cdot m y$, it grows more than quadratically when CONTACT is used. Thus, improving the accuracy of CONTACT would be of even greater value. This will be investigated in our future research.

Open Access This article is distributed under the terms of the Creative Commons Attribution Noncommercial License which permits any noncommercial use, distribution, and reproduction in any medium, provided the original author(s) and source are credited.

\section{References}

1. Shackleton P, Iwnicki SD (2007) Comparison of wheel-rail contact codes for railway vehicle simulation: an introduction to the Manchester Contact Benchmark and initial results. Veh Syst Dyn 46(1-2):129-149

2. Hertz H (1882) Über die Berührung fester elastischer Körper. Journal für reine und agewandte Mathematik 92:156-171

3. Shabana AA, Zaazaa KE, Sugiyama H (2008) Railroad Vehicle Dynamics: A Computational Approach. CRC Press, Boca Raton

4. Ayasse JB, Chollet H (2005) Determination of the wheel rail contact patch for semi-Hertzian conditions. Veh Syst Dyn 43:159-170

5. Kik W, Piotrowski J (1996) A fast approximate method to calculate normal load at contact between wheel and rail and creep forces during rolling. In: Zobory I (ed) Proceedings of the second Mini Conference on Contact mechanics and Wear of Wheel/Rail systems, Budapest

6. Ayasse JB, Chollet H (2006) Wheel-rail contact. In: Iwnicki SD (ed) Handbook of railway vehicle dynamics. CRC Press, Boca Raton, pp 85-120

7. Kalker JJ (1990) Three-dimensional elastic bodies in rolling contact. Solid mechanics and its applications. Kluwer Academic Publishers, Dordrecht

8. Kalker JJ (1979) The computation of three-dimensional rolling contact with dry friction. Int J Numer Methods Eng 14:1293-1307

9. Vollebregt EAH (2009) User's guide for CONTACT, J.J. Kalker's variational contact model. Technical Report TR09-03, VORtech. http://www.kalkersoftware.org

10. Kalker JJ (1982) A fast algorithm for the simplified theory of rolling contact. Veh Syst Dyn 11:1-13

11. Kalker JJ (1973) Simplified theory of rolling contact. Delft Prog Rep Ser C1 1:1-10 
12. Alonso A, Giménez JG (2007) Non-steady state modelling of wheel-rail contact problem for the dynamic simulation of railway vehicles. Veh Syst Dyn 46(3):179-196

13. Hou K, Kalousek J, Lamba H, Scott RT (2000) Thermal effect on adhesion in wheel/rail interface. In: Proceedings of the fifth international conference on contact mechanics and wear of rail/wheel systems, pp 239-244

14. Tomberger C, Dietmaier P, Sextro W, Six K (2009) Friction in wheel-rail contact: a model comprising interfacial fluids, surface roughness and temperature. In: Bracciali A (ed) Proceedings of the 8th international conference on contact mechanics and wear of rail/wheel systems, Firenze, pp 121-132

15. Dirks B, Enblom R (2009) Predition model for wheel profile wear and rolling contact fatigue. In: Bracciali A (ed) Proceedings of the 8th international conference on contact mechanics and wear of rail/wheel systems, Firenze, pp 935-943

16. Ziefle M, Nackenhorst U (2008) Numerical techniques for rolling rubber wheels: treatment of inelastic material properties and frictional contact. Comput Mech 42:337-356

17. Alonso A, Giménez JG (2007) Tangential problem solution for non-elliptical contact areas with the FastSim algorithm. Veh Syst Dyn 45(4):341-357

18. Giménez JG, Alonso A, Gómez E (2005) Introduction of a friction coefficient dependent on the slip in the Fastsim algorithm. Veh Syst Dyn 43:233-244

19. Piotrowski J (2010) Kalker's algorithm Fastsim solves tangential contact problems with slip-dependent friction and friction anisotropy. Veh Syst Dyn. doi:10.1080/00423110903178495

20. Shen ZY, Li Z (1996) A fast non-steady state creep force model based on the simplified theory. Wear 191:242-244
21. Vollebregt EAH, Iwnicki SD, Xie G, Shackleton P (2010) Assessing the accuracy of different simplified frictional rolling contact algorithms. Veh Syst Dyn (submitted). Available as Memo EV/M10.035, VORtech, Delft

22. Courant R, Hilbert D (1962) Methods of Mathematical Physics, Volume II. Wiley-Interscience, London

23. Boussinesq J (1885) Application des Potentiels à l'Étude de l'Équilibre et du Movement des Solides Élastiques. Paris, Gauthier-Villars

24. Cerruti V (1882) Ricerche intorno all'equilibrio dei corpi elastici isotropi. Reale Accademia dei Lincei, 13

25. Vollebregt EAH (1995) A Gauss-Seidel type solver for special convex programs, with application to frictional contact mechanics. J Optim Theor Appl 87(1):47-67

26. Piotrowski J, Kik W (2007) A simplified model of wheel/rail contact mechanics for non-Hertzian problems and its application in rail vehicle dynamics simulations. Veh Syst Dyn 46:27-48

27. Johnson KL (1985) Contact Mechanics. Cambridge University Press, Cambridge

28. Winkler E (1867) Die Lehre von der Elastizitaet und Festigkeit. Prag, Verlag von H. Dominicus

29. Kalker JJ (1967) On the rolling contact of two elastic bodies in the presence of dry friction. PhD thesis, Delft University of Technology, Delft

30. Heath MT (2002) Scientific Computing: An Introductory Survey, 2nd edn. McGraw-Hill, New York

31. Vollebregt EAH (2009) Refinement of Kalker's rolling contact model. In: Bracciali A (ed) In: Proceedings of the 8th international conference on contact mechanics and wear of rail/wheel systems, Firenze, pp 149-156 